\title{
A comparative analysis of senescence in adult damselfies and dragonflies (Odonata)
}

\author{
T. N. SHERRATT*, C. HASSALL*, R. A. LAIRD†, D. J. THOMPSON $\$$ \& \\ A. CORDERO-RIVERA§ \\ *Department of Biology, Carleton University, Ottawa, ON, Canada \\ †Department of Biological Sciences, University of Lethbridge, Lethbridge, AB, Canada \\ $\$$ School of Biological Sciences, University of Liverpool, Liverpool, UK \\ §Departamento de Ecoloxía e Bioloxía Animal, Universidade de Vigo, EUET Forestal, Pontevedra, Galicia, Spain
}

\author{
Keywords: \\ ageing; \\ mortality; \\ Odonata; \\ phylogeny; \\ sex; \\ territoriality.
}

\begin{abstract}
Any population whose members are subject to extrinsic mortality should exhibit an increase in mortality with age. Nevertheless, the prevailing opinion is that populations of adult damselflies and dragonflies do not exhibit such senescence. Here, we challenge this contention by fitting a range of demographic models to the data on which these earlier conclusions were based. We show that a model with an exponential increase in age-related mortality (Gompertz) generally provides a more parsimonious fit than alternative models including age-independent mortality, indicating that many odonates do indeed senesce. Controlling for phylogeny, a comparison of the daily mortality of 35 odonate species indicates that although male and female mortalities are positively correlated, mortality tends to be higher in males of those species that exhibit territoriality. Hence, we show for the first time that territoriality may impose a survivorship cost on males, once the underlying phylogenetic relationships are accounted for.
\end{abstract}

\section{Introduction}

Actuarial senescence ('ageing') is one of the most universal properties of organisms and is characterized by a population-level increase in rates of mortality and/or decrease in fecundity with chronological age (see Partridge \& Gems, 2002; Hughes \& Reynolds, 2005; Bonsall, 2006; Williams et al., 2006; Sherratt \& Wilkinson, 2009 for reviews). Age-dependent increases in mortality have now been reported in natural populations of a range of vertebrates (for a review see BrunetRossinni \& Austad, 2006), including mammals (e.g. Promislow, 1991; Gaillard et al., 2003) and birds (Ricklefs, 1998; Reed et al., 2008). Although the detection of senescence is particularly challenging in short-lived species with high overall rates of mortality (Hayflick, 2000; Kirkwood \& Austad, 2000), increases in agespecific mortality have nevertheless been reported recently in a variety of natural populations of short-lived insects (antler flies Protopiophila litigata (Bonduriansky),

Correspondence: Tom N. Sherratt, Department of Biology, Carleton University, 1125 Colonel By Drive, Ottawa, ON, Canada K1S 5B6. Tel.: +1 613520 2600, ext. 1748; fax: +1 613520 2569;

e-mail: sherratt@ccs.carleton.ca
Bonduriansky \& Brassil, 2002, 2005; neriid flies Telostylinus angusticollis (Enderlein), Kawasaki et al., 2008; honeybees Apis mellifera (L.), Dukas, 2008; crickets Teleogryllus commodus (Walker), Zajitschek et al., 2009a).

Evolutionary theory predicts that population-level accumulation of deleterious mutations (Medawar, 1952), trade-offs between early- and late-life performance (Williams, 1957; Kirkwood, 1977), and decelerating selection for somatic repair (Gavrilov \& Gavrilova, 2001; Laird \& Sherratt, 2009) should each lead to a form of actuarial senescence. However, the precise form that senescence takes in any system may be dependent on the specific mechanisms involved. For example, although human mortality curves are Gompertzian (exponential) in form (Gompertz, 1825), the simplest versions of reliability theory (Gavrilov \& Gavrilova, 2001) predict an initial near-Weibull (power) mortality function with an upper plateau. By contrast, evolutionary theories such as antagonistic pleiotropy (Williams, 1957) can only explain latelife asymptotic plateaus in mortality rates with some difficulty (Pletcher \& Curtsinger, 1998; Wachter, 1999). Therefore, characterizing the shape of the senescence curves in wild populations may help to elucidate the general proximate and ultimate mechanisms responsible. 
Comparing senescence patterns among taxonomically related species can also be enlightening. For example, several broad cross-species comparisons have revealed that overall rates of mortality within any given species can be shaped by the mating strategies that have evolved. In particular, Promislow (1992) found that the degree of male bias in adult mortality in mammals was positively correlated with the degree of sexual size dimorphism. Similarly, Liker \& Szekely (2005) conducted a phylogenetically controlled analysis of sex-specific adult mortality over 194 bird species and found that evolutionary increases in male-male competition were associated with male-biased mortalities.

Adult odonates (damselflies and dragonflies) are good candidates for investigating actuarial senescence in the field, because they are large enough to be marked individually and they can be re-sighted without the need for recapture (Cordero-Rivera \& Stoks, 2008). Mating systems also differ dramatically among odonate species, with males of some species exhibiting high territoriality and males of other species exhibiting little or no territoriality, allowing one to compare mortality rates in very different contexts. Until recently however, the prevailing opinion was that odonates do not show actuarial senescence (e.g. Banks \& Thompson, 1985; Fincke, 1986; Hamilton \& Montgomerie, 1989; Bennett \& Mill, 1995; Cordero-Rivera \& Stoks, 2008). This conclusion may have been somewhat premature however, as the vast majority of the above studies have based their conclusions simply on a qualitative assessment of the linearity of the logarithmic survivorship plots. Even those studies that have evaluated the fits of a wide range of different demographic models to odonate mark-recapture data using quantitative model selection criteria (e.g. Andres \& Andrés \& Cordero-Rivera, 2001; Anholt et al., 2001) have only rarely attempted to any fit demographic models that allow for age-dependent changes in mortality. Analysing an intensive mark-recapture study of the damselfly Coenagrion puella (L.) conducted over two separate years, Sherratt et al. (2010) recently fitted such models and in so doing provided strong evidence of senescence in a damselfly under field conditions. The question remains, however, whether many more examples of senescence in adult odonates have gone undetected, or whether this example of senescence in C. puella remains the exception rather than the rule. Here, we begin by fitting a range of demographic models to the log-survivorship plots depicted in a range of different mark-recapture studies of adult odonates, testing whether models with agedependent mortality provide a superior fit to the data than models with age-independent mortality.

The data we have collated have also allowed us to evaluate for the first time the implications of territoriality for overall differences in the rates of mortality between the sexes. Previous studies in a range of taxa such as lizards (e.g. Vanhooydonck et al., 2005; Lailvaux \& Irschick, 2007) indicate that male-male interactions in territorial species are more likely to escalate to fighting than in nonterritorial species. Likewise, there is ample evidence that territorial male damselflies and dragonflies aggressively interact with one another in an energetically and physically demanding way, to an extent rarely seen in nonterritorial males. For example, as Corbet (1999, p. 449 and citations within) reports although territorial male Calopteryx rarely fight directly, they frequently physically collide during territorial disputes, and they often can sustain damage to their wings and legs. In territorial libellulids, male territorial disputes can sometimes involve direct attacks including ramming and physical fighting including biting. Our prior expectation was that territoriality would impose a relative cost on males in terms of reduced survivorship compared to females. This prediction was in part influenced by the anticipated greater energetic and physical demands imposed by maintaining a territory. However, our prediction was also motivated by the observation that many nonterritorial damselfly species exhibit female-limited polymorphism, a phenomenon that is believed to have arisen in response to excessive harassment of females by males (Van Gossum et al., 2008). As male harassment may well lead to a decline in female survivorship (Córdoba-Aguilar, 2009), the opportunities for harassment generated by lack of male territoriality may act to lower the survival rates of females compared to males.

\section{Methods}

\section{Fitting of demographic models}

We used VistaMetrix ${ }^{\circledR}$ (http://www.skillcrest.com) to extract cumulative survivorship data from the semi-log plots (log numbers alive or log proportion alive vs. time since marking) depicted in a range of different markrecapture studies (summarized in Appendix S1). In all cases, we attempted to take readings to the highest level of precision possible. Studies involving an initial marked sample size of $<50$ mature adult individuals of a given sex were not considered (for example, ruling out Bennett \& Mill, 1995 with $n=24$ and 18 males and females, respectively). The semi-log plots were derived indirectly from the data presented by Pajunen (1962) and Tsubaki et al. (1997). Figure 1 shows the semi-log plots we derived (points) as well as the best-fitting models (continuous curves). We have complemented our analyses of extracted data with a quantitative analysis of the original tabular data of recaptures of males of Pyrrhosoma nymphula (Sulzer) (Corbet, 1952).

We used the demographic analysis software WINMoDEST 1.0.2 (Pletcher, 1999) to identify the mortality model that most closely fits the observed mortality patterns and to estimate the corresponding model parameters (see Min \& Tatar, 2006; Styer et al., 2007 for recent examples of this approach). Demographic models often represent death rates as the age-specific hazard (or instantaneous 

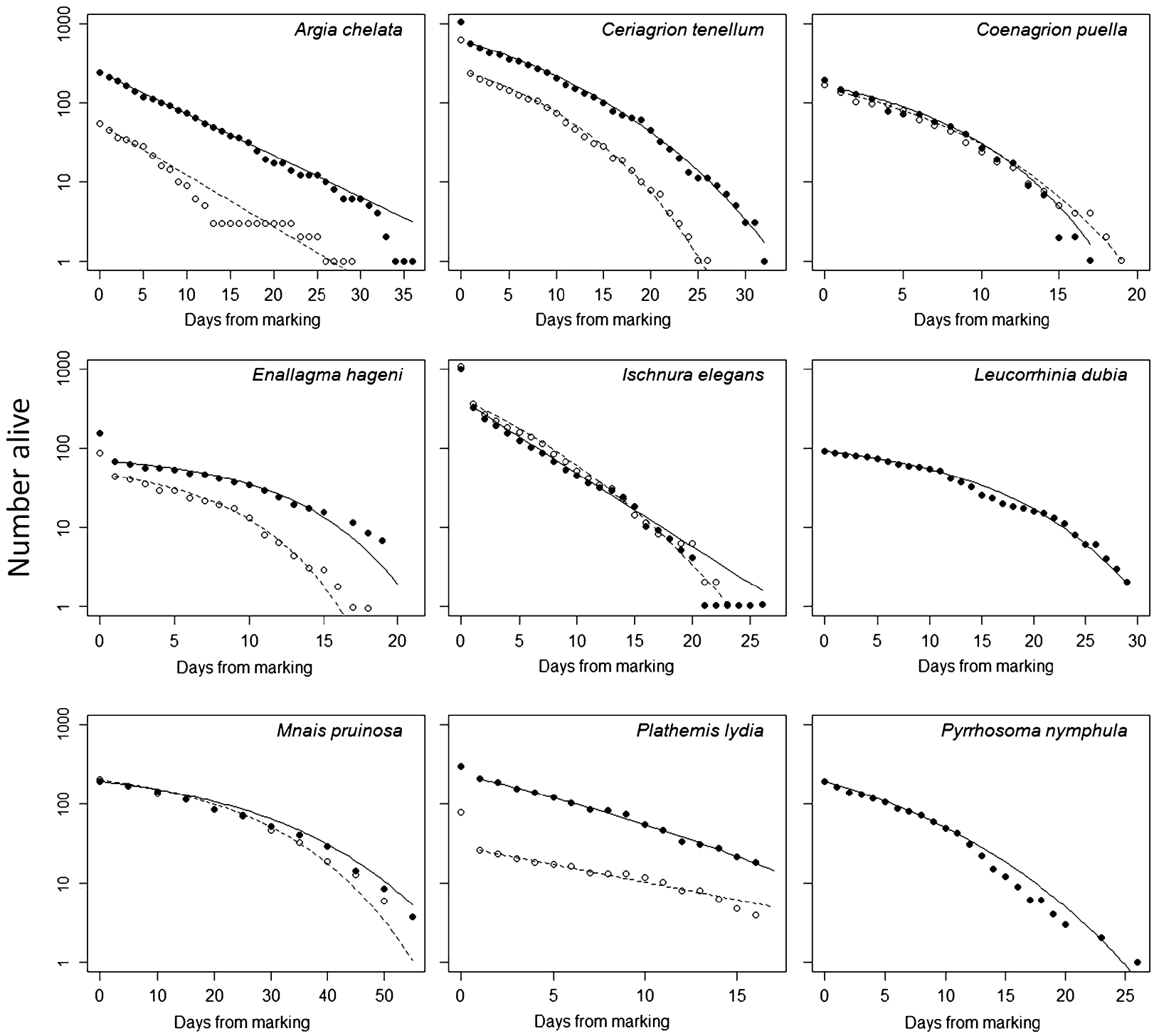

Fig. 1 Semi-log plots depicting the cumulative decline in the logarithm of the number of marked individuals alive, as reported in a variety of mark-recapture studies on damselflies and dragonflies. See Table 2 for original data sources. If daily rates of mortality were independent of age, then these plots should be strictly linear, reflecting a constant proportion of individuals lost from the population each time step. Dark filled circles represent males, whereas open circles represent females (with the exception of the data for Mnais pruinosa where filled circles represent orange-winged males and open circles represent clear-winged males). Fitted lines (solid, males; dashed, females) show the best fits of the most parsimonious model (Gompertz or no senescence) as translated to the cumulative survivorship curve (where $l_{x}=\exp \left(-\int \mu_{i} d i\right)$ so that $l_{x}=\exp \left\{\left(-\left(\frac{a}{b}\right)(\exp (b x)-1)\right\}\right.$ for Gompertz and $l_{x}=\exp (-a x)$ for no senescence), with best fit parameters given in Table $2 .{ }^{0}$

risk of failure) rather than the discrete-time probability of death (Gavrilov \& Gavrilova, 1991; Lee, 1992). An estimate of this instantaneous rate of mortality $\mu_{x}$ at age $x$ is approximated by:

$$
\mu_{x}=-\ln \left(\frac{N_{x+1}}{N_{x}}\right)
$$

where $N_{x+1} / N_{x}$ estimates the fraction of the population alive at age $x$ that also survives to age $x+1$. Note that if a constant fixed proportion of the marked population is unavailable for re-sighting on any given day, then eqn (1) still estimates hazard rate, as the ratio of recaptures is unchanged.

The WinModest 1.0.2 software fits four inter-related models (Table 1) that may account for some of the observed changes in the age-specific hazard (see Fig. 2). Estimates of the parameters of each model are derived by maximum likelihood estimation (MLE), and the most appropriate model chosen on the basis of log-likelihood (the less negative the log-likelihood, the better the fit). 
Table 1 Five inter-related hazard functions (age-specific hazard rate) and their associated probability density of time to death $[f(x)]$. The corresponding likelihood of observing a given distribution pattern of time to death is $\prod^{n} f\left(x_{i}\right)$ where $x_{i}$ is the time interval in

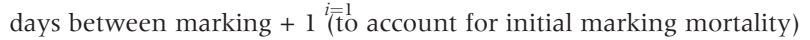
and last recapture for the $i^{\text {th }}$ individual, and $n$ is the size of the marked population re-sighted on at least day 1 following marking. Symbols: $x$, age; $\mu_{x}$, age-specific hazard rate; $a$, initial mortality rate; $b$, exponential rate of increase with age; $c$, age-independent mortality; $s$, rate of mortality deceleration. For ease of presentation, we define the function $\omega(x)$ where $\omega(x)=1+\left(\frac{a s}{b}\right)\left(e^{b x}-1\right)$.

\begin{tabular}{lll}
\hline Model & $\begin{array}{l}\text { Hazard } \\
\text { function }\end{array}$ & $\begin{array}{l}\text { Probability density of } \\
\text { time to death } f(x)\end{array}$ \\
\hline No senescence & $\mu_{x}=a$ & $a e^{-a x}$ \\
Gompertz & $\mu_{x}=a e^{b x}$ & $a \exp \left[b x-\frac{a}{b}\left(e^{b x}-1\right)\right]$ \\
Gompertz-Makeham & $\mu_{x}=a e^{b x}+c$ & $\left(c+a e^{b x}\right) \exp \left[-\frac{a}{b}\left(e^{b x}-1\right)-c x\right]$ \\
Logistic & $\mu_{x}=a e^{b x} \omega(x)^{-1}$ & $a e^{b x} \omega(x)^{-(s+1) / s}$ \\
Logistic-Makeham & $\mu_{x}=a e^{b x} \omega(x)^{-1}$ & $e^{c x} \omega(x)^{-1 / s}\left(a e^{b x} \omega(x)^{-1}+c\right)$ \\
& $+c$ & \\
\hline
\end{tabular}

As the fitted models are partially nested, we used likelihood ratio tests (Hilborn \& Mangel, 1997) to evaluate the effectiveness of models of gradually increasing complexity in parsimoniously explaining the demographic patterns. Of the four candidate models, the Gompertz equation simply assumes that mortality starts at rate $a$ and increases with age at exponential rate $b$. Adding a constant $c$ to the Gompertz equation introduces a fixed rate of age-independent death - this model is known as the Gompertz-Makeham model. Some demographic studies have found that at very late ages, mortality rates decelerate and may even reach an upper plateau (Carey et al., 1992; Vaupel et al., 1998). The logistic model allows for such a sigmoidal change in mortality rate with age, yet setting the rate of mortality deceleration $s$ to zero recovers the Gompertz. Likewise, adding a constant $c$ to the logistic model introduces a source of age-independent 'extrinsic' mortality to this sigmoidal relationship: this model is known as the logistic-Makeham model. A fifth model, a single parameter 'no senescence' model, simply assumes that mortality rates are constant throughout an individual's lifetime. Because WinModest does not fit the null model of no senescence, we fitted the no senescence model based on our own MLE routines, developed in MathCad ${ }^{\circledR} v 12$ (PTC, Needham, MA, USA). Unfortunately in one field study (Koenig \& Albano, 1985), the fate of the oldest 5\% of individuals were not depicted, so in this case the parameter estimates of the Gompertz and no senescence models were based on fitting the appropriate cumulative survivorship curves by nonlinear least squares (NLLS) (Ricklefs \& Scheuerlein, 2002).

Two features are immediately apparent from the semilog plots we have collated (Fig. 1). First, many of the plots exhibit a marked decline in apparent survivorship the day after marking (see Cordero-Rivera \& Stoks,
2008), which may arise as a consequence of slight damage during the marking process and/or as a consequence of stress-induced dispersal (Cordero, 1994; Cordero-Rivera \& Stoks, 2008). To allow for these effects, we decided at the outset to look for evidence of agedependent mortality starting the day after the first day of marking.

Second, the semi-log plots at each time step show, as might be expected, an increase in variance as the size of the surviving population diminishes. Although qualitative evidence of senescence is sometimes based on directly regressing age-specific mortality against age (see for example Hughes \& Charlesworth, 1994; Stearns \& Kaiser, 1996), the regression method alone is not a reliable test of senescence, because it does not incorporate information on the variances of estimated probabilities of survival (Slade, 1995). One potential solution to the problem is to conduct a standard linear regression of hazard rate vs. age, yet weight the hazard rate by the square root of the number alive at age $x$ (Promislow, 1991). This weighting is appropriate because the standard error of binomially distributed data is proportional to $1 / \sqrt{ } n$ where $n$ is the sample size. Exploratory MonteCarlo simulations covering population sizes and mortality rates typical of the above odonate examples (Appendix S2) show that although weighted linear regressions can produce slightly higher than the usual permissible type I error rate (rejection of $H_{0}$ of no senescence when true), they are considerably more reliable than unweighted regressions on the same data. As a first screening, we have therefore cautiously applied weighted linear regression models to the data to test for significant changes of hazard with age. Strictly speaking eqn (1) is only valid if the length of the time intervals is large enough to include at least one observed death. Therefore, to be conservative, our direct estimates of hazard were not derived once the population ever reached such a small size that there was no change in the numbers alive on consecutive days. Despite all of these precautions, the weighted regression method simply provides a transparent alternative approach to evaluating the strength of evidence for senescence. Our primary method of analysis was that of MLE coupled with log-likelihood ratio tests, because it is generally acknowledged that this approach involves less bias (Mueller et al., 1995; Pletcher, 1999; Nussey et al., 2008).

\section{Differences in overall mortality between species}

Although odonate populations may potentially show age-related increases in mortality, the overall average mortality (and hence longevity) of cohorts of odonates may differ between sexes of the same species and among species. Here, we analyse data collated by Cordero-Rivera \& Stoks (2008) detailing the estimated daily survival rate of males and females based on 53 mark-recapture studies of 35 species (Appendix S3). Although territoriality is 

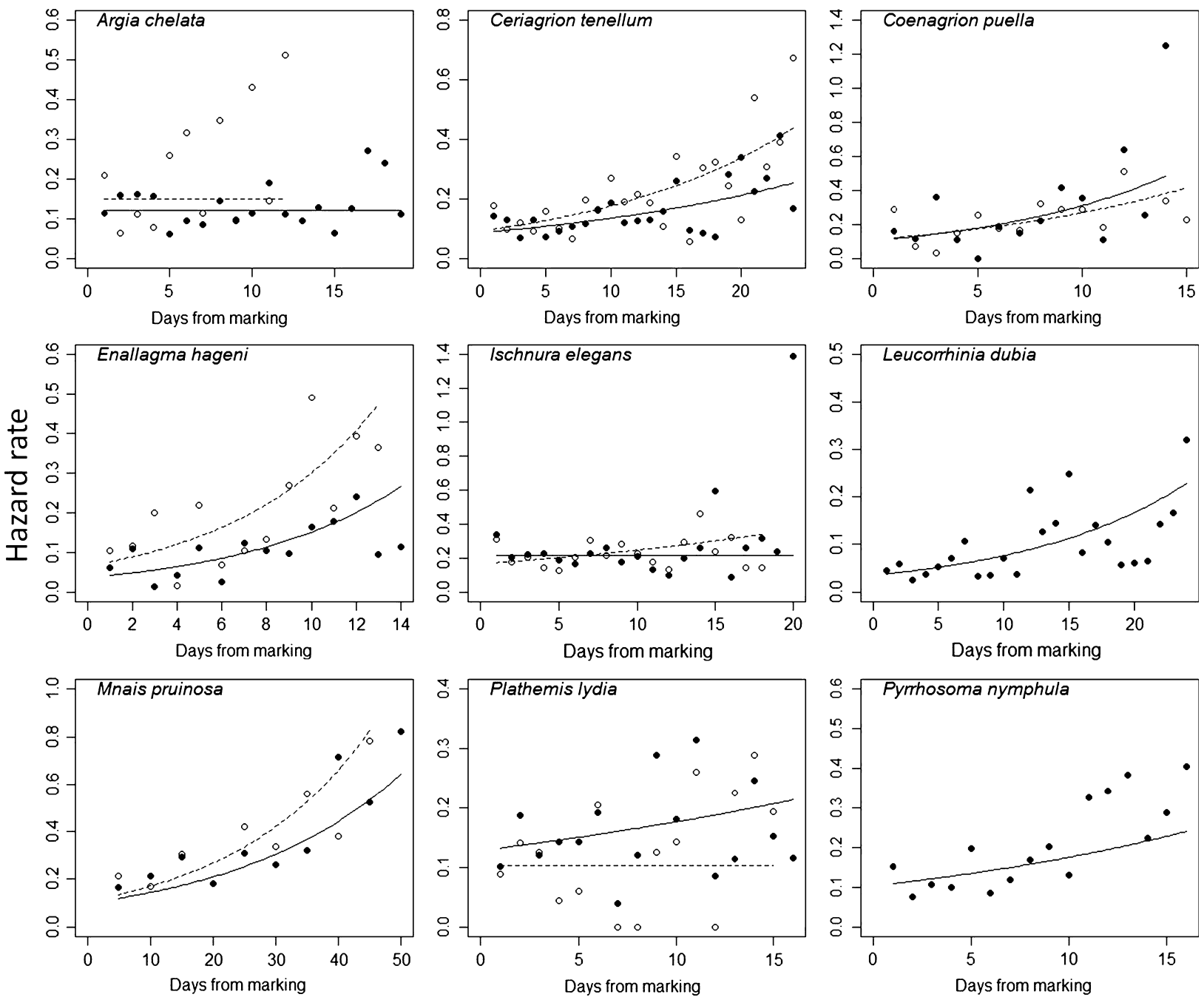

Fig. 2 Estimated hazard rate [-log (proportion of population surviving) for each time step], derived from the semi-log plots for each species. Hazard rates were not calculated from marking to day 1, thereby excluding all marked individuals that were never seen again. Moreover, the estimates of hazard depicted do not always extend the full duration of the relevant study because we stopped hazard estimation once the population had reached such a small size that there was no change in the numbers alive on consecutive days. Fitted lines (solid, males; dashed, females) show the best fits of the most parsimonious hazard model (Gompertz or no senescence; see Table 1 for hazard functions), with best fit parameters given in Table 2.

something of a continuum - for example the coenagrionid $P$. nymphula is only weakly territorial in adult stage we simply classified each species as territorial or nonterritorial, using Cordero-Rivera \& Stoks (2008) descriptions, which were assigned without regard to mortality rates.

To test for patterns in differential rates of mortality between the sexes, we conducted a cross-species comparison of male mortality (= 1-estimated daily survivorship), employing the arithmetic mean mortality when multiple estimates had been made. Because mortality rates may potentially co-vary with mean species body size, we also included mean male abdomen length (in $\mathrm{mm})$, using data compiled from a variety of sources
(Appendix S3). Our first analysis involved fitting a General Linear Model (GLM Type III, simultaneous sums of squares) with the presence/absence of male territoriality as a fixed factor and male size and female mortality as covariates, as well as a female mortality $\times$ territoriality interaction to allow the gradient of the fitted relationship between female and male mortality to differ, dependent on whether the species was territorial or nonterritorial.

One key issue of particular concern in the above sets of analysis is the potential lack of independence of species data through shared phylogeny. For example, male Calopteryx japonica (Selys) and male Calopteryx virgo (L.) are both territorial damselflies, and both species appear to exhibit higher male mortality than female mortality, yet 
(a)

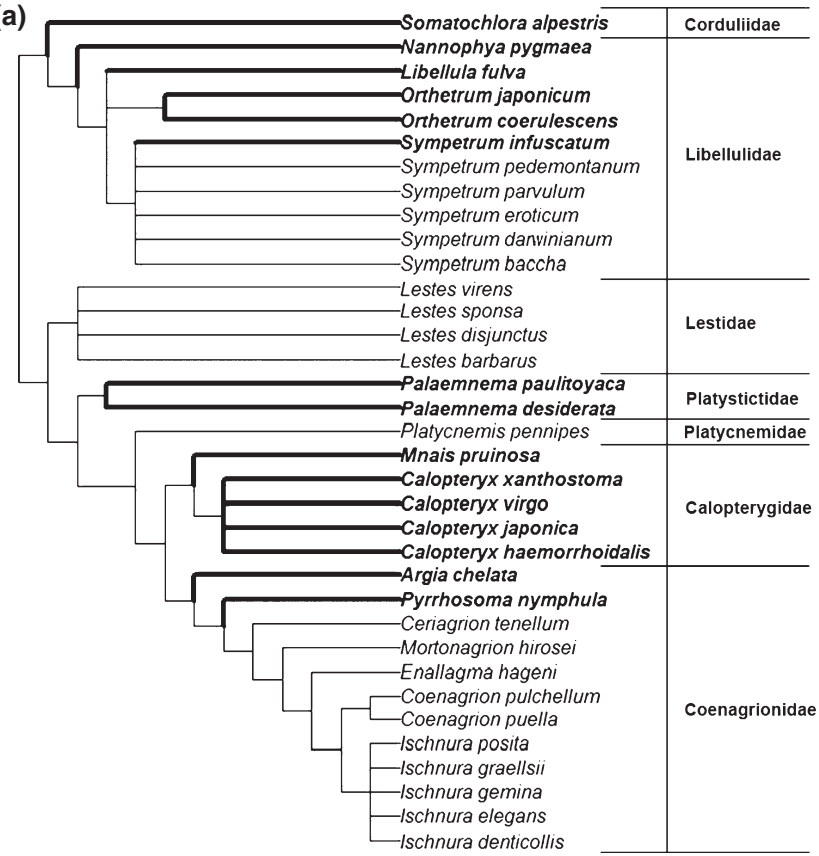

(b)

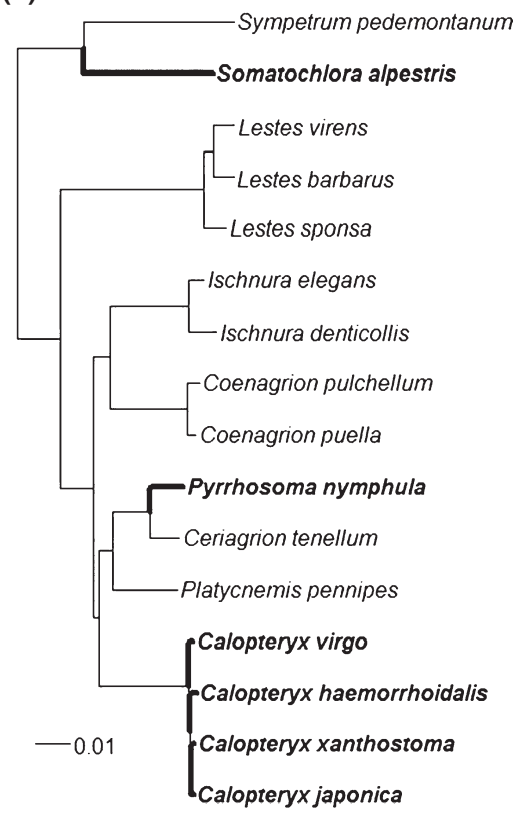

Fig. 3 (a) Cladogram showing the presence of territoriality (in bold) in the 35 odonate species for which survivorship data are available; phylogenetic relationships based on O'Grady \& May, 2003; Ware et al., 2007; Rehn, 2003 and (b) phylogeny of 16 species for which comparable sequences are available showing territoriality (in bold).

the pairing of mortality and territoriality traits may be ancestral, so it is questionable whether they should be treated as providing independent data points (Harvey \& Pagel, 1991). An additional set of analyses which attempted to control for the phylogenetic effects was therefore performed.

Because of the absence of a well-supported phylogeny for the 35 species, a full phylogenetically controlled analysis was not possible. In its place, we present three complementary analyses. The first two methods account for hierarchical levels of classification but either ignore or make simplifying assumptions about, the true phylogenetic distances. The third method (based on a reduced data set) incorporates current estimates of phylogenetic differences. Our first two approaches analysed the attributes of all 35 species in the review by Cordero-Rivera $\&$ Stoks (2008). The assumed phylogenetic relationships underlying these two analyses were based on O'Grady \& May (2003), Rehn (2003) and Ware et al. (2007). Figure 3a shows how territoriality maps onto this phylogenetic tree and demonstrates that territoriality is common on some branches of the phylogeny but not others. In our first attempt to control for phylogeny, male mortality, male size, female mortality and territoriality were entered separately as response variables in ANovas with genus nested within family, nested within suborder. Using the resulting residuals for each variable, we fitted the model 'male mortality $=$ territoriality + male size + female mortality + female mortality $\times$ territoriality'.
Although this approach does not account for all phylogenetic autocorrelation, it does ameliorate the problem (Purvis, 2008). Our second approach fitted the same model to the same phylogeny, this time using the comparative generalized estimating equation function 'compar.gee' in the APE package (Paradis et al., 2004; Freckleton, 2009) in R (R Development Core Team., 2009) while making the simplifying assumption that the branch lengths were consistently 1 .

Our third analysis was restricted to 16 species (six territorial and ten nonterritorial) for which comparable genetic sequences were available (18S rRNA+5.8S rRNA+28S rRNA, see Appendix S4, Table S3 for GenBank accession numbers). Sequences were aligned using MEGA4 (Tamura et al., 2007) and phylogenies constructed using the neighbour-joining method and 1000 bootstrap replicates. The model 'male mortality $=$ territoriality + male size + female mortality + female mortality $\times$ territoriality' was again fitted to the resulting (reduced) phylogeny (Fig. 3b) using 'compar.gee' in the APE package (Paradis et al., 2004).

\section{Results}

Both our comparisons of the fits of candidate demographic models and our weighted linear regression analyses indicated that a significant majority of odonate species surveyed exhibit significant increases in mortality with time from first marking (Table 2). Hazard rates do 


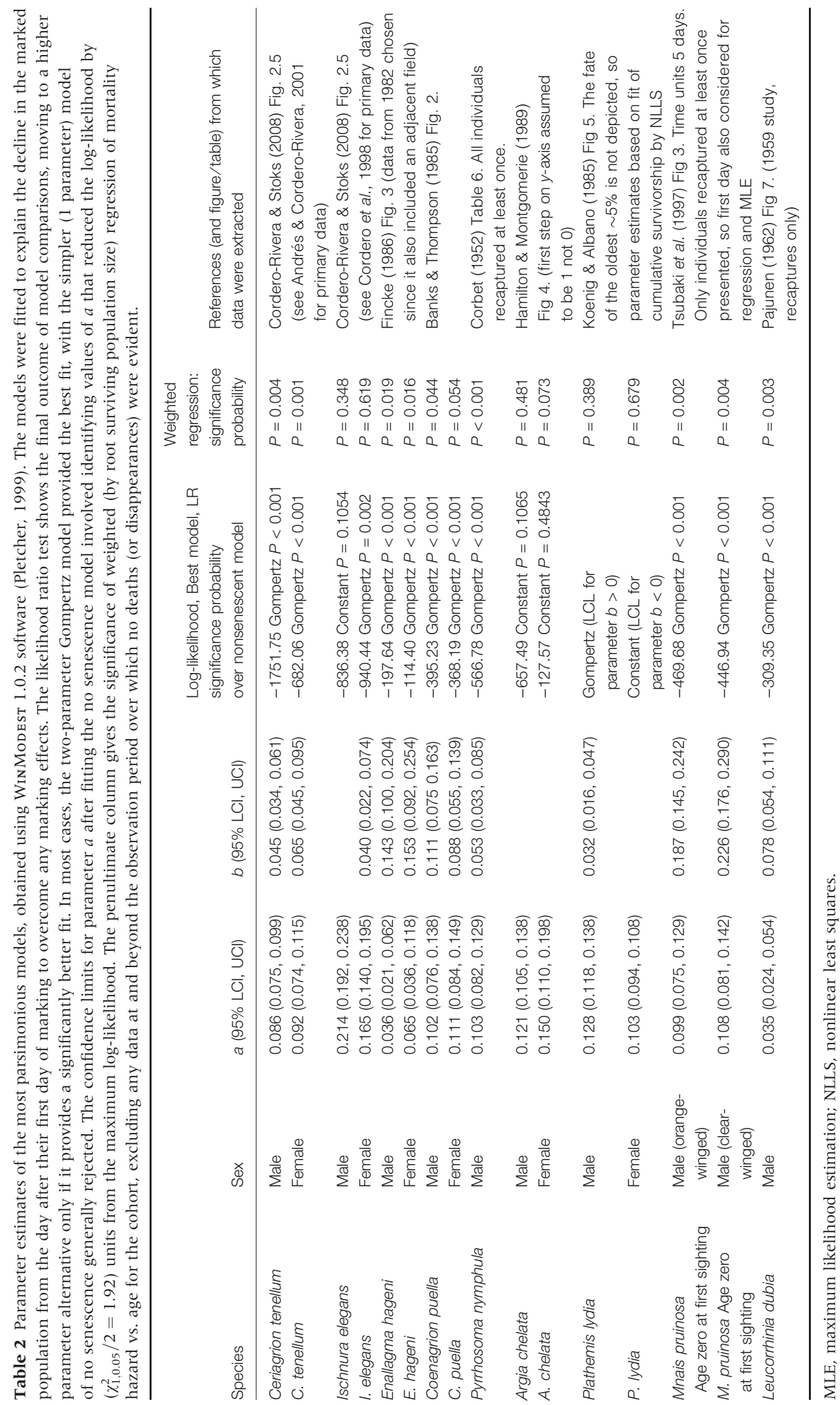


indeed appear to increase with age in many species (Fig. 2). The three exceptions to this pattern were the damselflies Ischnura elegans, Argia chelata and the dragonfly Plathemis lydia - the available data on these species do not allow us to unequivocally reject the null hypothesis of no senescence. As might be expected, these particular species have the lowest estimated exponential coefficient $b$ after fitting the Gompertz - so small that inclusion of the parameter does not improve the fit (Table 2). Intriguingly, across all of the cases examined (with the exception of P. lydia where parameters were necessarily estimated using NLLS), there was a significant negative correlation between the MLE estimates of $a$ and $b(r=-0.526$, d.f. $=14, P=0.036$ after fitting Gompertz to the additional three cases where no senescence represented the most parsimonious model; $r=-0.367$, d.f. $=11, P=0.218$ without these three cases), suggesting that populations with high initial mortality exhibited little sign of ageing, whereas those with low initial mortality show more rapid ageing.

Prior to phylogenetic control, male mortality was significantly positively related to female mortality (see Fig. 4), but not male abdomen size (see Table 3 for all significance tests). There was no significant effect of territoriality on male mortality, and the female mortality $\times$ territoriality interaction was not significant. Using the nested ANova as a method for phylogenetic control, male mortality was significantly positively related to female mortality and positively related to territoriality, but abdomen size was not significant and neither was the female mortality $\times$ territoriality interaction. Very similar results (highlighting the significance of territoriality as a main effect increasing male mortality when controlling for phylogeny in the nested model) were obtained when the dependent variable was the continuity-corrected estimate of mortality, male hazard [ $=-\ln$ (daily survivorship)]. Moreover, when we fitted a phylogenetically controlled model to the entire phylogeny using the GEE method and assuming fixed branch lengths of 1, territoriality was consistently significant at least as part of the interaction, whether the dependent variable was male mortality or hazard. When the comparative GEE method was used on the restricted data set for which phylogenetic distances could be estimated, there were no significant relationships between male mortality and male size, female mortality, territoriality and the female mortality $\times$ territoriality interaction (Table 3 ).

\section{Discussion}

We fitted a range of models to the log-survivorship plots of a range of odonate species, and concluded, in contrast

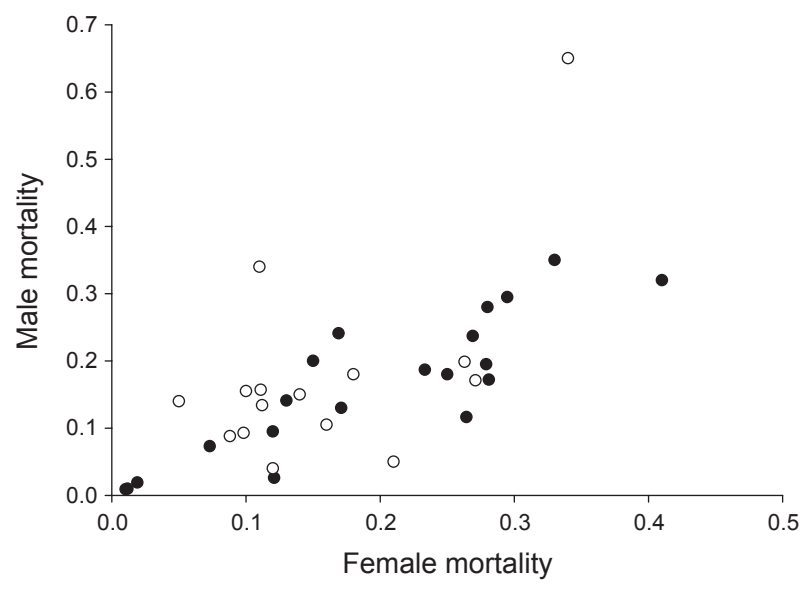

Fig. 4 The relationship between male and female daily mortality in 35 species of damselfly and dragonfly (data from Cordero-Rivera $\delta$ Stoks, 2008). Open circles represent species with territorial males, whereas the closed circles represent species with nonterritorial males.

Table 3 The results of our comparative analyses of male mortality, not controlling for phylogeny and controlling for it in 3 separate ways. In each case, a model with male abdomen size, female mortality, male territoriality and a female mortality $\times$ territoriality interaction was fitted. The degrees of freedom for the first two methods of analysis were consistently 1 and 30 . For the simplified fixed branch length tree ( 35 species), the 'phylogenetic degrees of freedom' were estimated as 13.48. For the reduced tree with estimated branch lengths (16 species), the phylogenetic degrees of freedom were 6.25 .

\begin{tabular}{|c|c|c|c|c|}
\hline \multirow[b]{2}{*}{ Dependent variable } & \multicolumn{4}{|l|}{ Method of analysis } \\
\hline & GLM (no control) & Nested ANOVA & Fixed branch length tree & Reduced tree \\
\hline \multicolumn{5}{|l|}{ Male daily mortality } \\
\hline Male abdomen size & $F=0.6621, P=0.422$ & $F=1.8938, P=0.179$ & $t=1.285, P=0.233$ & $t=-4.629, P=0.098$ \\
\hline Female mortality & $F=19.6499, P<0.001$ & $F=33.3371, P<0.001$ & $t=8.439, P<0.001$ & $t=1.634, P=0.311$ \\
\hline Territoriality & $F=1.070, P=0.309$ & $F=16.4076, P<0.001$ & $t=-0.918, P=0.013$ & $t=-0.355, P=0.773$ \\
\hline \multicolumn{5}{|l|}{ Male hazard } \\
\hline Male abdomen size & $F=0.7314, P=0.399$ & $F=2.1048, P=0.1572$ & $t=1.522, P=0.164$ & $t=-4.729, P=0.095$ \\
\hline Female hazard & $F=12.5494, P=0.001$ & $F=31.4971, P<0.001$ & $t=7.219, P<0.001$ & $t=1.540, P=0.329$ \\
\hline Territoriality & $F=1.0689, P=0.309$ & $F=31.0213, P<0.001$ & $t=-1.757, P=0.115$ & $t=-0.420, P=0.735$ \\
\hline Female hazard $\times$ territoriality & $F=3.8019, P=0.061$ & $F=0.010, P=0.921$ & $t=4.339, P=0.002$ & $t=1.030, P=0.462$ \\
\hline
\end{tabular}


to the conclusions drawn in earlier papers, that the majority of these studies exhibit a degree of actuarial senescence (Table 2). Thus, adult odonates should no longer be treated as anomalous with respect to senescence. These conclusions were generally robust whatever the methodology we applied to detect senescence whether it was through weighted regressions of daily hazard rates, or through the fits (and comparisons) of demographic models via maximum likelihood. Indeed, an alternative method of estimating model parameters this time by NLLS fitted directly to the cumulative survivorship plots - produced qualitatively identical results (Appendix S5, Figs S2a,b). Corbet (1952) had previously tentatively proposed that the results of his mark-recapture analysis of $P$. nymphula were consistent with a survival rate which decreases with age, and our re-analysis of the data he presented confirms this suspicion. Not only do the estimates of hazard tend to increase with time since marking (Fig. 2), but the downward curvature of a number of logarithmic survivorship plots is evident (Fig. 1), which in itself is indicative of an increase in mortality with advancing age. This downward curvature is evident in a number of logarithmic survivorship plots on odonates in addition to the ones analysed here, including the dragonfly Sympetrum danae (Sulzer) under natural and semi-natural conditions (Fig. 4 of Michiels \& Dhondt, 1989) and a range of damselfly species reared under laboratory conditions (Fig. 1 of Cordero, 1994). However, the specific census points in each of these cases were not depicted, so they were not analysed.

Of course, with the exception of Corbet (1952), all of our data were gleaned from published graphs as opposed to the raw data. Nevertheless, our method of data extraction was highly repeatable in that when the same set of data was independently extracted by two different researchers the two data sets were highly correlated $(r>0.99$ for all tested combinations of species, sex and morph). More importantly, our approach necessarily involved treating the entire population of marked individuals as a single cohort, even though they were marked on a range of days. The 'single cohort' approach not only reflects the standard way that odonate survivorship curves have been generated and interpreted, but it has been extensively employed in previous studies on senescence in wild-caught insects (Bonduriansky \& Brassil, 2002; Dukas, 2008; Kawasaki et al., 2008) on the basis that the prevailing weather conditions at the time of the study were sufficiently uniform to allow combining data in this way. Reassuringly, our earlier analyses of the demography of the damselfly C. puella (Sherratt et al., 2010) yielded quantitatively similar results, whether the data were treated as a single cohort or not.

The papers from which we extracted data generally refer to reproductively active (mature) individuals, but a second factor that may add significant noise is uncer- tainty over the individual's age on first marking. Although one might argue that: (i) adult age should be measured from the time maturity is reached, and (ii) any new visitors to the water body will tend to be recently mature individuals, the fact remains that the exact adult age on first marking is not known. Uncertainty over the precise age will no doubt add noise, but (as we have seen) it is unlikely to confound our analysis because actual age is likely to correlate well with time since first marking. Crespin et al. (2006) describe a simulation model that supports the validity of this approximation, whereas our own analysis of senescence in C. puella of known age (marked as newly emerged tenerals) yielded similar conclusions to those in an analysis of a larger cohort of C. puella at unknown age at time of marking (Sherratt et al., 2010). Although innovative methods now exist to evaluate senescence in populations in which subjects are of unknown age at the time of marking (Müller et al., 2004; Zajitschek et al., 2009b), these approaches necessarily assume a fixed population density and stable age structure, which are unfortunately invalid in odonates with seasonal emergence patterns.

Field studies have a special role to play in understanding senescence, not least to ensure that laboratory results are not just artefacts of laboratory conditions. For example, Linnen et al. (2001) showed that wild-caught Drosophila lived for as long as those in an inbred laboratory Drosophila line under selection for longevity for nearly 20 years. Although the overall age-dependent increases in hazard were consistently best explained by a simple exponential rise, the selection of Gompertz model may be as much a function of its simplicity as the goodness of fit it provides. Of the range of recent laboratory studies of insect demography which use Pletcher's (1999) WinModest software to fit and compare a range of mortality curves, there are a number of examples that also find Gompertzian mortality (e.g. Iliadi et al., 2009; Maklakov \& Fricke, 2009; Scannapieco et al., 2009), whereas others have found logistic mortality (e.g. Bilde et al., 2009; Gomez et al., 2009; Burger et al., 2010), so it is clear that our finding of Gompertz is not entirely a consequence of high extrinsic rates of mortality and low sample sizes associated with field conditions. Likewise, it is not known why I. elegans, $A$. chelata and $P$. lydia show no evidence of senescence whereas other species do, although it is clear that it is not a question of the sample size of marked individuals alone, because populations in several studies employing smaller sample sizes exhibited senescence.

Intriguingly, the Gompertzian parameters $a$ and $b$ were negatively correlated among species when the entire data set was considered. Ricklefs \& Scheuerlein (2002) suggest that when deaths arise early in an organism's life, then they are likely to arise as a result of 'extrinsic' factors such as predation, whereas those deaths that arise late in an organism's life are much more likely to arise owing to 'intrinsic' factors, such as wing-wear. The authors used 
this observation to propose that increases in extrinsic mortality could effectively mask intrinsically driven mortality. One possible mechanism that may enhance the effect is condition-dependent mortality, with populations exhibiting high early mortality effectively purged of members of the population with weak constitution.

As a consequence, Ricklefs \& Scheuerlein (2002) suggest that $b$ should decrease to compensate for an increase in $a$, thereby maintaining an approximately constant age-dependent component of mortality $(\sqrt{ }\{a b\})$. We have found some evidence for this phenomenon here, although only when species with low age-dependent exponents $(b)$ were included in the analysis.

Given the paucity of data on senescence in odonates, and the lack of analyses for age effects, our comparative analyses of the effects of territoriality were necessarily restricted to analysing overall daily mortality rather than senescence (a change in daily mortality) per se. We found that the mortality rates of male and female odonate species were strongly positively correlated across species. Part of this relationship may be attributed to shared environment of the two sexes (because mortality is likely to vary even among populations of the same species), but it is likely that it also reflects an underlying robustness of individual species. Controlling for female mortality, there was no significant effect of territoriality on male mortality when phylogenetic relationships were ignored. However, when analysing the survivorship patterns of the 35 species, we found a significant effect of territoriality on male mortality in the anticipated direction (territoriality increasing male mortality rates after controlling for female mortality), once the underlying phylogenetic relationships were accounted for. Our analysis of a much smaller data set (16 species) using the estimated phylogenetic distances did not detect such a pattern, but the low sample size inevitably reduces the power of this test.

Why should territoriality act to reduce male survivorship relative to female survivorship? As previously noted, territoriality might expose males to increased damage caused by male-male conflict whereas nonterritoriality might expose females to increased damage caused by male harassment. This raises the further question of why natural selection does not make territorial males (and females in nonterritorial systems) more robust, to fully deal with their higher damage rates. Classical evolutionary theory suggests that, over generations, direct, extrinsic reductions in male relative survivorship could result in the evolution of males of territorial species also having intrinsically reduced survivorship compared to males of nonterritorial species (Medawar, 1952; Williams, 1957 but see Abrams, 1993). Recent work in evolutionary reliability theory also suggests that where there is decelerating selection for robustness against damage, natural selection will typically 'under-compensate' for this damage (Laird \& Sherratt, 2009), again predicting that territorial males should also be intrinsically more likely to have reduced survivorship. Intriguingly, in
Mnais pruinosa costalis (Selys), territorial male morphs appear to have shorter lives than nonterritorial male morphs, even under laboratory conditions (Tsubaki et al., 1997), suggesting that patterns of mortality are not simply a direct reflection of extrinsic mortality.

Whereas this current study has focused on survivorship over the reproductive stage of mature adult odonates, previous work has examined how territoriality might influence differential activity patterns and survivorship of males and females prior to maturation (Anholt et al., 1991; Anholt, 2008). In particular, whereas females of a range of odonate species increase in mass between emergence and maturity by an average of $125 \%$, only males of territorial species exhibit a comparable increase (Anholt et al., 1991), possibly as a consequence of their relatively high energy demands (see also Anholt, 2008). So, whereas some of the costs of territoriality may well arise before reproductive maturity, here we have some evidence to suggest that territorial males pay at least some of the price for their behaviour postreproduction.

Cross-species patterns in mortality as a consequence of sexual selection have previously been reported in other taxonomic groups, although not so far in the context of territoriality. For example, Promislow et al. (1992) found that the differences in mortality rates between the sexes of different species of passerine birds increased with male plumage brightness (see also Promislow et al., 1994). The findings of this current study not only challenge the view that odonates are too short lived to exhibit senescence, but also indicate that their survivorship patterns are shaped by a combination of intra-sexual and inter-sexual conflict (Svensson \& Sheldon, 1998).

\section{Acknowledgments}

We thank Carlos Esquivel and Enrique González Soriano for providing data on the body sizes of several species in our data set. Our Associate Editor Rhonda Snook, and two anonymous referees provided some very helpful critiques and advice. We are grateful to NSERC (TNS \& RAL), the Government of Ontario (CH), NERC - NE/ C511205/1 (DJT) and the Spanish Ministry of Science and Innovation (CGL2008-02799) for funding.

\section{References}

Abrams, P.A. 1993. Does increased mortality favor the evolution of more rapid senescence? Evolution 47: 877-887.

Andrés, J.A. \& Cordero-Rivera, A. 2001. Survival rates in a natural population of Ceriagrion tenellum (Villers) (Odonata): effects of sex and female phenotype. Ecol. Entomol. 26: 341346.

Anholt, B.R. 2008. Fitness landscapes, mortality schedules, and mating systems. In: Dragonflies and Damselflies: Model Organisms for Ecological and Evolutionary Research (A. Córdoba-Aguilar, ed.), pp 175-188. Oxford University Press, Oxford. 
Anholt, B.R., Marden, J.H. \& Jenkins, D.M. 1991. Patterns of mass gain and sexual dimorphism in adult dragonflies (Insecta: Odonata). Can. J. Zool. 69: 1156-1163.

Anholt, B.R., Vorburger, C. \& Knaus, P. 2001. Mark-recapture estimates of daily survival rates of two damselflies (Coenagrion puella and Ischnura elegans). Can. J. Zool. 79: 895-899.

Banks, M.J. \& Thompson, D.J. 1985. Lifetime mating success in the damselfly Coenagrion puella. Anim. Behav. 33: 1175-1183.

Bennett, S. \& Mill, P.J. 1995. Pre- and post-maturation survival in adults of the damselfly Pyrrhosoma nymphula (Zygoptera: Coenagrionidae). J. Zool. 235: 559-575.

Bilde, T., Maklakov, A.A., Meisner, K., la Guardia, L. \& Friberg, U. 2009. Sex differences in the genetic architecture of lifespan in a seed beetle: extreme inbreeding extends male lifespan. BMC Evol. Biol. 9: 33.

Bonduriansky, R. \& Brassil, C.E. 2002. Rapid and costly ageing in wild male flies. Nature 420: 377.

Bonduriansky, R. \& Brassil, C.E. 2005. Reproductive ageing and sexual selection on male body size in a wild population of antler flies (Protopiophila litigata). J. Evol. Biol. 18: 1332-1340.

Bonsall, M.B. 2006. Longevity and ageing: appraising the evolutionary consequences of growing old. Philos. Trans. $R$ Soc. Lond. B 361: 119-135.

Brunet-Rossinni, A.K. \& Austad, S.N. 2006. Senescence in wild populations of mammals and birds. In: Handbook of the Biology of Aging (E.J. Masoro \& S.N. Austad, eds), pp. 243-266. Academic Press, Burlington, MA.

Burger, J.M.S., Buechel, S.D. \& Kawecki, T.J. 2010. Dietary restriction affects lifespan but not cognitive aging in Drosophila melanogaster. Aging Cell 9: 327-335.

Carey, J.R., Liedo, P., Orozco, D. \& Vaupel, J.W. 1992. Slowing of mortality rates at older ages in large medfly cohorts. Science 258: $457-461$.

Corbet, P.S. 1952. An adult population study of Pyrrhosoma nymphula (Sulzer): (Odonata: Coenagrionidae) J. Anim. Ecol. 21: 206-222.

Corbet, P.S. 1999. Dragonflies: Behaviour and Ecology of Odonata. Harley Books, Colchester.

Cordero, A. 1994. The effect of sex and age on survivorship of adult damselflies in the laboratory (Zygoptera: Coenagrionidae). Odonatologica 23: 1-12.

Cordero, A., Santolamazza Carbone, S. \& Utzeri, C. 1998. Mating opportunities and mating costs are reduced in androchrome female damselflies, Ischnura elegans (Odonata). Anim. Behav. 55: 185-197.

Cordero-Rivera, A. \& Stoks, R. 2008. Mark-recapture studies and demography. In: Dragonflies and Damselflies: Model Organisms for Ecological and Evolutionary Research (A. Córdoba-Aguilar, ed.), pp. 7-20. Oxford University Press, Oxford.

Córdoba-Aguilar, A. 2009. A female evolutionary response when survival is at risk: male harassment mediates early reallocation of resources to increase egg number and size. Behav. Ecol. Sociobiol. 63: 751-763.

Crespin, L., Harris, M.P., Lebreton, J.-D. \& Wanless, S. 2006. Increased adult mortality and reduced breeding success with age in a population of common guillemot Uria aalge using marked birds of unknown age. J. Avian Biol. 37: 273-282.

Dukas, R. 2008. Mortality rates of honey bees in the wild. Insect. Soc. 55: 252-255.

Fincke, O.M. 1986. Lifetime reproductive success and the opportunity for selection in a nonterritorial damselfly (Odonata: Coenagrionidae). Evolution 40: 791-803.
Freckleton, R.P. 2009. The seven deadly sins of comparative analysis. J. Evol. Biol. 22: 1367-1375.

Gaillard, J.-M., Loison, A., Festa-Bianchet, M., Yoccoz, N.G. \& Solberg, E. 2003. Ecological correlates of lifespan in populations of large herbivorous mammals. In: Life Span: Evolutionary, Ecological, and Demographic Perspectives (J.R. Carey \& S. Tuljapurkar, eds), pp. 39-56. Population Council, New York.

Gavrilov, L.A. \& Gavrilova, N.S. 1991. The Biology of Life Span: A Quantitative Approach. Harwood Academic Publishers, Chur, Switzerland.

Gavrilov, L.A. \& Gavrilova, N.S. 2001. The reliability theory of aging and longevity. J. Theor. Biol. 213: 527-545.

Gomez, F.H., Bertoli, C.I., Sambucetti, P., Scannapieco, A.C. \& Norry, F.M. 2009. Heat-induced hormesis in longevity as correlated response to thermal-stress selection in Drosophila buzzatii. J. Therm. Biol. 34: 14-22.

Gompertz, B. 1825. On the nature of the function expressive of the law of human mortality, and on the mode of determining the value of life contingencies. Philos. Trans. R Soc. Lond. B 115 513-585.

Hamilton, L.D. \& Montgomerie, R.D. 1989. Population demography and sex ratio in a neotropical damselfly (Odonata: Coenagrionidae) in Costa Rica. J. Trop. Ecol. 5: 159-171.

Harvey, P.H. \& Pagel, M.D. 1991. The Comparative Method in Evolutionary Biology. Oxford University Press, Oxford.

Hayflick, L. 2000. The future of ageing. Nature 408: 267-269.

Hilborn, R. \& Mangel, M. 1997. The Ecological Detective: Confronting Models with Data. Princeton University Press, Princeton, NJ.

Hughes, K.A. \& Charlesworth, B. 1994. A genetic analysis of senescence in Drosophila. Nature 367: 64-66.

Hughes, K.A. \& Reynolds, R.M. 2005. Evolutionary and mechanistic theories of aging. Annu. Rev. Entomol. 50: 421-445.

Iliadi, K.G., Iliadi, N.N. \& Boulianne, G.L. 2009. Regulation of Drosophila life-span: effect of genetic background, sex, mating and social status. Exp. Gerontol. 44: 546-553.

Kawasaki, N., Brassil, C.E., Brooks, R.C. \& Bonduriansky, R. 2008. Environmental effects on the expression of life span and aging: an extreme contrast between wild and captive cohorts of Telostylinus angusticollis (Diptera: Neriidae). Am. Nat. 172 346-367.

Kirkwood, T.B.L. 1977. Evolution of ageing. Nature 270: 301304.

Kirkwood, T.B.L. \& Austad, S.N. 2000. Why do we age? Nature 408: $233-238$.

Koenig, W.D. \& Albano, S.S. 1985. Patterns of territoriality and mating success in the white-tailed skimmer Plathemis lydia (Odonata: Anisoptera). Am. Midl. Nat. 114: 1-12.

Lailvaux, S.P. \& Irschick, D.J. 2007. The evolution of performance-based male fighting ability in Caribbean Anolis lizards. Am. Nat. 170: 573-586.

Laird, R.A. \& Sherratt, T.N. 2009. The evolution of senescence through decelerating selection for system reliability. J. Evol. Biol. 22: 974-982.

Lee, E.T. 1992. Statistical Methods for Survival Data Analysis. John Wiley and Sons, New York.

Liker, A. \& Szekely, T. 2005. Mortality costs of sexual selection and parental care in natural populations of birds. Evolution 59: 890-897.

Linnen, C., Tartar, M. \& Promislow, D. 2001. Cultural artifacts: a comparison of senescence in natural, laboratory-adapted and artificially selected lines of Drosophila melanogaster. Evol. Ecol. Res. 3: $877-888$ 
Maklakov, A.A. \& Fricke, C. 2009. Sexual selection did not contribute to the evolution of male lifespan under curtailed age at reproduction in a seed beetle. Ecol. Entomol. 34: 638643.

Medawar, P.B. 1952. An Unsolved Problem of Biology. Western Printing Systems, Bristol.

Michiels, N.K. \& Dhondt, A.A. 1989. Effects of emergence characteristics on longevity and maturation in the dragonfly Sympetrum danae (Anisoptera: Libellulidae). Hydrobiologia 171: 149-158.

Min, K.-J. \& Tatar, M. 2006. Restriction of amino acids extends lifespan in Drosophila melanogaster. Mech. Ageing Dev. 127: 643646.

Mueller, L.D., Nusbaum, T.J. \& Rose, M.R. 1995. The Gompertz equation as a predictive tool in demography. Exp. Gerontol. 30: 553-569.

Müller, H.-G., Wang, J.-L., Carey, J.R., Caswell-Chen, E.P., Chen, C., Papadopoulos, N. \& Yao, F. 2004. Demographic window to aging in the wild: constructing life tables and estimating survival functions from marked individuals of unknown age. Aging Cell 3: 125-131.

Nussey, D.H., Coulson, T., Festa-Bianchet, M. \& Gaillard, J.-M. 2008. Measuring senescence in wild animal populations: towards a longitudinal approach. Funct. Ecol. 22: 393-406.

O'Grady, E.W. \& May, M.L. 2003. A phylogenetic reassessment of the subfamilies of Coenagrionidae (Odonata: Zygoptera). J. Nat. Hist. 37: 2807-2834.

Pajunen, V.I. 1962. Studies on the population ecology of Leucorrhinia dubia V. d. Lind. (Odon., Libellulidae). Ann. Zool. Soc. "Vanamo" 24: 1-79.

Paradis, E., Claude, J. \& Strimmer, K. 2004. APE: analyses of phylogenetics and evolution in R language. Bioinformatics 20: 289-290.

Partridge, L. \& Gems, D. 2002. The evolution of longevity. Curr. Biol. 12: R544-R546.

Pletcher, S.D. 1999. Model fitting and hypothesis testing for agespecific mortality data. J. Evol. Biol. 12: 430-439.

Pletcher, S.D. \& Curtsinger, J.W. 1998. Mortality plateaus and the evolution of senescence: why are old age mortality rates so low? Evolution 52: 454-464.

Promislow, D.E.L. 1991. Senescence in natural populations of mammals - a comparative study. Evolution 45: 1869-1887.

Promislow, D.E.L. 1992. Costs of sexual selection in natural populations of mammals. Proc. $R$ Soc. Lond. B 247: 203-210.

Promislow, D.E.L., Montgomerie, R. \& Martin, T.E. 1992. Mortality costs of sexual dimorphism in birds. Proc. $R$ Soc. Lond. B 250: 143-150.

Promislow, D., Montgomerie, R. \& Martin, T.E. 1994. Sexual selection and survival in North American waterfowl. Evolution 48: 2045-2050.

Purvis, A. 2008. Phylogenetic approaches to the study of extinction. Annu. Rev. Ecol. Evol. Syst. 39: 301-319.

R Development Core Team. 2009. R: A Language and Environment for Statistical Computing. R Foundation for Statistical Computing, Vienna, Austria.

Reed, T., Kruuk, L.E.B., Wanless, S., Frederiksen, M., Cunningham, E.J.M. \& Harris, M.P. 2008. Reproductive senescence in a long-lived seabird: rates of decline in late life performance are associated with varying costs of early reproduction. Am. Nat. 171: E89-E101.

Rehn, A.C. 2003. Phylogenetic analysis of higher-level relationships of Odonata. Syst. Entomol. 28: 181-239.
Ricklefs, R.E. 1998. Evolutionary theories of aging: confirmation of a fundamental prediction, with implications for the genetic basis and evolution of life span. Am. Nat. 152: 24-44.

Ricklefs, R.E. \& Scheuerlein, A. 2002. Biological implications of the Weibull and Gompertz models of aging. J. Gerontol. 57A: B69-B76.

Scannapieco, A.C., Sambucetti, P. \& Norry, F.M. 2009. Direct and correlated responses to selection for longevity in Drosophila buzzatii. Biol. J. Linn. Soc. 97: 738-748.

Sherratt, T.N. \& Wilkinson, D.M. 2009. Big Questions in Ecology and Evolution. Oxford University Press, Oxford.

Sherratt, T.N., Laird, R.A., Hassall, C., Lowe, C.D., Harvey, I.F., Watts, P.C. et al. 2010. Empirical evidence of senescence in adult damselflies (Odonata: Zygoptera). J. Anim. Ecol. 79: 1034-1044.

Slade, N.A. 1995. Failure to detect senescence in persistence of some grassland rodents. Ecology 76: 863-870.

Stearns, S.C. \& Kaiser, M. 1996. Effects on fitness components of P-element inserts in Drosophila melanogaster. Evolution 50: 795 806.

Styer, L.M., Carey, J.R., Wang, J.-L. \& Scott, T.W. 2007. Mosquitoes do senesce: departure from the paradigm of constant mortality. Am. J. Trop. Med. Hyg. 76: 111-117.

Svensson, E. \& Sheldon, B.C. 1998. The social context of life history evolution. Oikos 83: 466-477.

Tamura, K., Dudley, J., Nei, M. \& Kumar, S. 2007. mega4: Molecular Evolutionary Genetics Analysis (MEGA) software version 4.0. Mol. Biol. Evol. 24: 1596-1599.

Tsubaki, Y., Hooper, R.E. \& Siva-Jothy, M.T. 1997. Differences in adult and reproductive lifespan in the two male forms of Mnais pruinosa costalis Selys (Odonata: Calopterygidae). Res. Popul. Ecol. 39: 149-155.

Van Gossum, H., Sherratt, T.N. \& Cordero-Rivera, A. 2008. The evolution of sex-limited colour polymorphism. In: Dragonflies and Damselflies: Model Organisms for Ecological and Evolutionary Research (A. Córdoba-Aguilar, ed.), pp. 219-231. Oxford University Press, Oxford.

Vanhooydonck, B., Herrell, A.Y., Van Damme, R. \& Irschick, D.J. 2005. Does dewlap size predict male bite performance in Jamaican Anolis lizards? Funct. Ecol. 19: 38-42.

Vaupel, J.W., Carey, J.R., Christensen, K., Johnson, T.E., Yashin, A., Holm, N.V. et al. 1998. Biodemographic trajectories of longevity. Science 280: 855-860.

Wachter, K.W. 1999. Evolutionary demographic models for mortality plateaus. Proc. Natl Acad. Sci. USA 96: 10544-10547.

Ware, J., May, M.L. \& Kjer, K. 2007. Phylogeny of the higher Libelluloidea (Anisoptera: Odonata): an exploration of the most speciose superfamily of dragonflies. Mol. Phylogenet. Evol. 45: 289-310.

Williams, G.C. 1957. Pleiotropy, natural selection, and the evolution of senescence. Evolution 11: 398-411.

Williams, P.D., Day, T., Quinn, F. \& Rowe, L. 2006. The shaping of senescence in the wild. Trends Ecol. Evol. 21: 458-463.

Zajitschek, F., Bonduriansky, R., Zajitschek, S.R.K. \& Brooks, R. 2009a. Sexual dimorphism in life history: age, survival and reproduction in male and female field crickets Teleogryllus commodus under seminatural conditions. Am. Nat. 173: 792802.

Zajitschek, F., Brassil, C.E., Bonduriansky, R. \& Brooks, R. 2009b. Sex-effects on lifespan and senescence in the wild when dates of birth and death are unknown. Ecology 90: 16981707. 


\section{Supporting information}

Additional Supporting Information may be found in the online version of this article:

Appendix S1 Details of the nine mark-recapture studies that have been re-analysed to assess the strength of evidence for actuarial senescence.

Appendix S2 An evaluation of the Type I error rate associated with weighted and unweighted regressions of hazard vs. age.

Appendix S3 A summary of mark-recapture studies on 35 species of odonate.

Appendix S4 Accession numbers for sequences used in phylogenetic analysis.
Appendix S5 Comparison of parameter estimates from maximum likelihood and non-linear least squares.

As a service to our authors and readers, this journal provides supporting information supplied by the authors. Such materials are peer-reviewed and may be reorganized for online delivery, but are not copy-edited or typeset. Technical support issues arising from supporting information (other than missing files) should be addressed to the authors.

Received 1 September 2010; revised 8 December 2010; accepted 10 December 2010 